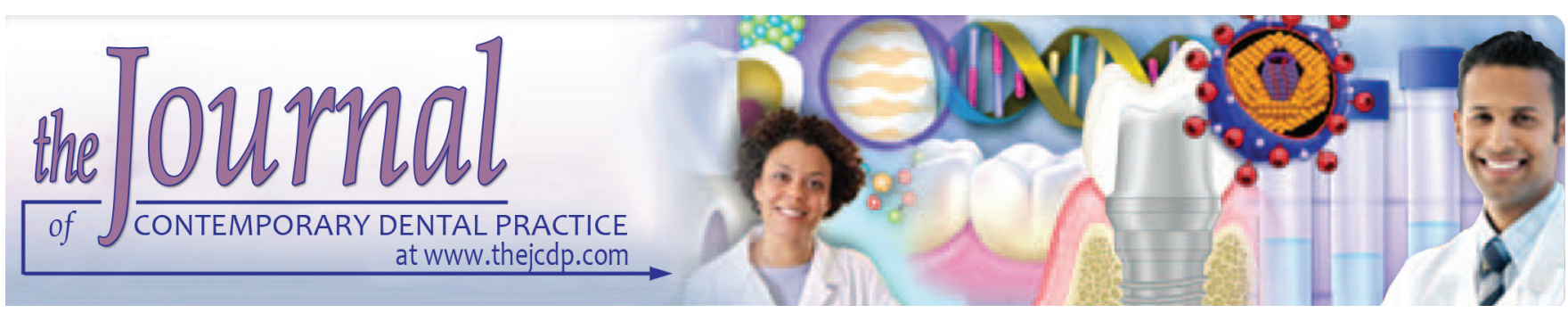

\title{
Effect of Tannic Acid on Bond Strength of Etch and Rinse and Self-etch Adhesive Systems in Dentin of Primary Teeth
}

\author{
${ }^{1}$ Masoumeh Ebrahimi, ${ }^{2}$ Alireza Sarraf Shirazi, ${ }^{3}$ Faraneh Abdolhoseinpour, ${ }^{4}$ Mojtaba Abdollahi
}

\begin{abstract}
Introduction: One of the possible mechanisms for the gradual destruction of bond strength in dentin-resin interface, could be due to the demineralized unstable collagen matrix. Use of protease inhibitors, such as tannic acid (TA) could prevent destruction of collagen fibers. The aim of this study was to compare the TA effect on bond strength of etch and rinse and self-etch adhesive systems in the dentin of primary teeth.
\end{abstract}

Materials and methods: This in vitro study was done on 40 extracted primary molar teeth. The teeth were sectioned in the mesiodistal direction, and enamel of buccal and lingual surfaces was removed. Samples were randomly divided into four groups: Single bond (SB) + TA, SB, Clearfil SE Bond (CSB) + TA, and CSB. Then, Z250 and Clearfil AP-X composites were cured on the surfaces of SB and CSB groups respectively. After that, all samples were divided into aging and non-aging groups. For 3 months, samples were placed under 1,000 thermal cycles in aging group. Subsequently, the shear bond strengths of all groups were measured by the International testing machine, and failure mode was evaluated by an optical stereomicroscope. Data were analyzed with paired t-test and independent t-test.

Results: Tannic acid induced a significant reduction in the immediate bond strength of adhesive SB. Meanwhile, TA had no significant effect on shear bond strength of the CSB system.

${ }^{1}$ Department of Pediatric Dentistry, Dental Research Center and Dental School of Mashhad University of Medical Sciences Mashhad, Islamic Republic of Iran

${ }^{2}$ Department of Pediatric Dentistry, Dental Research Center School of Dentistry, Mashhad University of Medical Sciences Mashhad, Islamic Republic of Iran

${ }^{3}$ Department of Pediatric Dentistry, School of Dentistry, Dental Branch, Islamic Azad University, Tehran, Islamic Republic of Iran

${ }^{4}$ Department of Pedodontics, Private Practice, Mashhad, Islamic Republic of Iran

Corresponding Author: Faraneh Abdolhoseinpour, Department of Pediatric Dentistry, School of Dentistry, Dental Branch, Islamic Azad University, Tehran, Islamic Republic of Iran, e-mail: faraneh.abdolhoseinpour@gmail.com
Conclusion: Based on our findings, use of TA is not recommended with SB and CSB adhesives on primary teeth.

Clinical significance: Tannic acid may not be considered in resin restorations of primary teeth.

Keywords: Bond strength, Etch and rinse adhesive systems, Primary teeth, Self-etch adhesive systems, Tannic acid.

How to cite this article: Ebrahimi M, Sarraf Shirazi A, Abdolhoseinpour F, Abdollahi M. Effect of Tannic Acid on Bond Strength of Etch and Rinse and Self-etch Adhesive Systems in Dentin of Primary Teeth. J Contemp Dent Pract 2017;18(1):34-38.

Source of support: Nil

Conflict of interest: None

\section{INTRODUCTION}

Nowadays, increase in dental awareness among parents has inclined them to maintain the primary teeth of their children. Therefore, tooth-colored restorations are highly regarded due to the need of maintaining the teeth structure and esthetics. ${ }^{1}$ This feature of restoration is a special issue for pediatric dentistry. Despite significant improvements in bond strength of dental materials with tooth structure, durability of bond strength is still questionable. ${ }^{2}$

In the past decade, several in vivo and in vitro studies have shown that the bond between dentin and resin materials would damage and finally weaken over time. ${ }^{3,4}$ Also, not all of these bondings could be applied without controversy or concern about biological safety. ${ }^{5}$

One of the possible mechanisms for the gradual destruction of bond could be the demineralized unstable collagen matrix. This matrix is prone to degradation via metalloproteinase enzymes of dentinal, salivary, or bacterial origin. ${ }^{6}$ Some studies have shown that use of protease inhibitors, such as chlorhexidine and tannic acid (TA) could prevent destruction of collagen fibers by making intermolecular or intramolecular cross-links within the collagen fibers. ${ }^{7,8}$ 
Tannic acid is a commercial form of tannin, a polyphenol with weak acidity. A recent study has shown that TA could bond by its hydroxyl group with amide $(\mathrm{NH})$ of collagen through hydrogen bonds. This bond would increase stability of the collagen fibers and elevate their resistance against dentin matrix metalloproteinases (MMPs). ${ }^{9}$

Therefore, due to lack of such studies in this field and by considering the importance of this issue, the present study aimed to investigate the effect of TA on bond strength of etch and rinse and self-etch adhesive systems in the dentin of primary teeth.

\section{MATERIALS AND METHODS}

In this in vitro study, the effect of TA was evaluated on bond strength of composite restorations of primary dentin. Forty freshly extracted primary molar teeth were collected and stored in $0.1 \%$ thymol solution. The sample size was calculated by PASS test in NCSS software $\alpha=0.05$.

By mesiodistal sectioning, all samples were divided into lingual and buccal halves, and finally, 80 specimens were obtained. This process was performed by a long needle diamond bur (Mani Japan) and a high-speed handpiece (NSK, Japan) with air-water spray. The enamel of buccal-lingual was removed by using a trimmer (Dentaurum, Germany). Then, to prepare a perfectly smooth surface of dentin, papers of 300,600, and 1200 grit of silicon carbide were used.

For evaluating the bond strength, samples were mounted in the following steps:

Each sample was placed on a glass slab from the smooth surface of dentin. Then, we put a piece of $5 \mathrm{cc}$ syringe on each prepared tooth to cover it. Following that the syringe has been filled with a self-cured acrylic resin (Fig. 1).

Due to lack of studies in this field and also for determining the best time for TA application, we performed a pilot study. The result of the pilot study showed that the

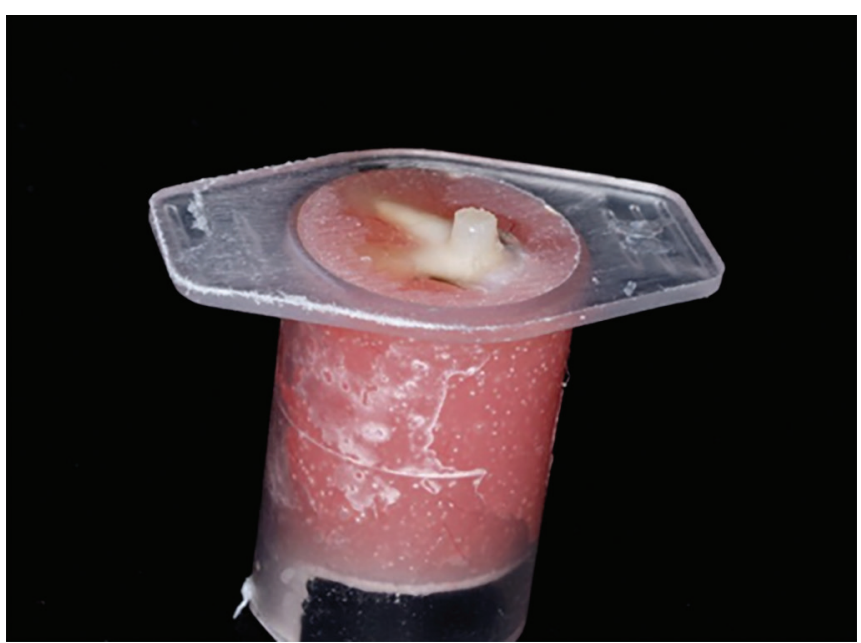

Fig. 1: A prepared sample best application times for TA were 120 and 30 seconds for Adper single bond (SB) (3M ESPE, St. Paul, MN, USA) and Clearfil SE bond (CSB) (Kuraray, Osaka, Japan) groups respectively.

Based on adhesive type, samples were randomly divided into two groups, A and B. TA and adhesives were applied to the buccal surface of each sample (subgroups $\mathrm{A} 1$ and B1) but $\mathrm{T}$ lingual surfaces were treated only with bondings (without TA) (subgroups A2 and B2).

\section{Subgroup A}

A1: Phosphoric acid (37\%) (Ultra-etch, Ultradent, USA) was used on dentin for 15 seconds and rinsed with water for 10 seconds. Excess water was dried with a cotton pellet then, TA ( $20 \%)$ was applied over the surface for 120 seconds. The excess of TA was then removed by gentle air drying. The SB adhesive was used in two layers and after using a mild stream of air, the curing was performed via a light cure unit (Bluephase, Ivocolar Vivadent, Austria) for 20 seconds at a intensity of $600 \mathrm{~mW} / \mathrm{cm}^{2}$.

Following this, the composite Z250 (3M, ESPE, USA) was condensed on the surface by using a mold with $2 \mathrm{~mm}$ in thick and $2 \mathrm{~mm}$ in diameter and was cured for 40 seconds (Fig. 1).

A2: All processes were performed similarly in subgroup A1 except that TA was not applied.

\section{Subgroup B}

B1: The dentin surface was treated by CSB primer according to the manufacturer's instructions. After mild stream of air, TA $(20 \%)$ was applied to the surface for 30 seconds. The CSB adhesive was applied and curing was done for 20 seconds. Following that, Clearfil AP-X composite was placed similar to the first group and cured for 40 seconds. $B 2$ : The procedure was the same as for the previous subgroup, but TA was not applied.

Then, samples of each group were divided randomly into two groups; the first group did not undergo the aging process. The second group was stored in saline and $37^{\circ} \mathrm{C}$ incubation for 3 months. After that, the specimens underwent 1,000 temperature cycles between 5 and $55^{\circ} \mathrm{C}$ with 20 seconds of dwell time. Then, shear bond strength and failure mode assessment were performed for all groups.

\section{Measurement of Shear Bond Strength}

The shear bond strength was measured by universal testing machine (Zwick, GmbH and Co., Ulm, Germany) at the dental and composite interface with $1 \mathrm{~mm} / \mathrm{min}$ rate.

\section{Failure Mode Evaluation}

The type of fraction was determined by an optical stereomicroscope (Dino-Lite, Taiwan) and categorized under 
three groups: Adhesive (fraction between bonding and teeth or composite), cohesive (fraction in teeth or composite), and mixed (combination of both types of failure modes).

\section{Statistical Analysis}

Statistical analysis was performed by Statistical Package for the Social Sciences (SPSS version 16). For analyzing data related to buccal and lingual surfaces of tooth with/ without TA, paired t-test was used; independent t-test was applied for data analysis of buccal and lingual surfaces of different teeth.

\section{RESULTS}

The TA effect on the bond strength of adhesive systems (etch and rinse and self-etch) in the dentin of primary teeth was evaluated in this study.

Kolmogorov-Smirnov test showed the normality of data distribution with a 5\% error level.

\section{Comparison of Bond Strengths}

The mean shear bond strength was significantly higher in CSB to SB in TA non-aging group. $(\mathrm{p}=0.001)$ (Table 1$)$. Also, the mean of shear bond strength in subgroup without TA was significantly more than subgroup with TA by using SB ( $\mathrm{p}=0.007)$ (Table 1 and Graph 1 ).

Table 1: Mean of shear bond strength between CSB and SB groups without aging

\begin{tabular}{llll}
\hline & \multicolumn{2}{c}{ Bonding } & \\
\cline { 2 - 3 } Tannic acid & SB (mean \pm SD) & CSB (mean \pm SD) & p-value \\
\hline Yes & $30.2 \pm 11.1$ & $70.68 \pm 16.23$ & 0.001 \\
No & $63.01 \pm 23.75$ & $60.39 \pm 18.91$ & \\
p-value & 0.007 & 0.26 & \\
\hline
\end{tabular}

SD: Standard deviation

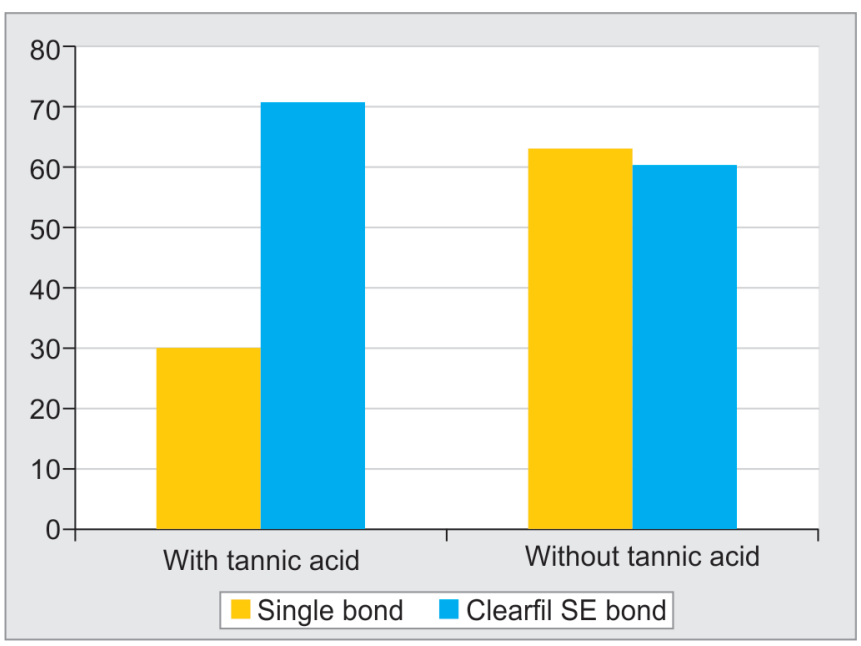

Graph 1: Mean shear bond strength of SB and CSB subgroups with/without TA in without aging group
The t-test was used for comparison of mean shear bond strength related to SB with TA in aging and nonaging groups. No significant differences were observed between groups $(\mathrm{p}=0.68)$.

The CSB had superiority to SB in the non-aging group $(p=0.001)$, and the mean shear bond strength of CBS was significantly higher in non-aging group compare to aging group ( $p=0.002$ ). In evaluating the relationship between mean of shear bond strength related to CSB subgroups, there was significant difference between aging and without aging groups ( $\mathrm{p}=0.92)$ (Table 2 and Graph 2 ).

The comparison of shear bond strength of SB and CSB subgroups in the aging control groups showed a significant difference $(p=0.001)$. However, in non-aging groups no significant difference was reported between subgroups ( $\mathrm{p}=0.193)$ (Tables 2 and 3$)$.

\section{Failure Mode Pattern}

The findings of fraction patterns are reported in three categories: Adhesive, cohesive, and mixed. Based on these data, failure mode of the adhesive was predominant in SB and CSB subgroups among both aging/non-aging and with/without using TA.

\section{DISCUSSION}

From the early introduction of adhesive systems up to now, they constantly changed due to enhancment of the

Table 2: Mean shear bond strength of SB and CSB subgroups with/without aging in TA group

\begin{tabular}{|c|c|c|c|}
\hline \multirow[b]{2}{*}{ Aging } & \multicolumn{2}{|c|}{ Bonding } & \multirow[b]{2}{*}{$p$-value } \\
\hline & $S B($ mean $\pm S D)$ & $C S B($ mean $\pm S D)$ & \\
\hline Yes & $33.38 \pm 21.41$ & $51.6 \pm 17.81$ & 0.053 \\
\hline No & $30.2 \pm 11.1$ & $70.68 \pm 16.23$ & 0.001 \\
\hline$p$-value & 0.68 & 0.022 & \\
\hline
\end{tabular}

SD: Standard deviation

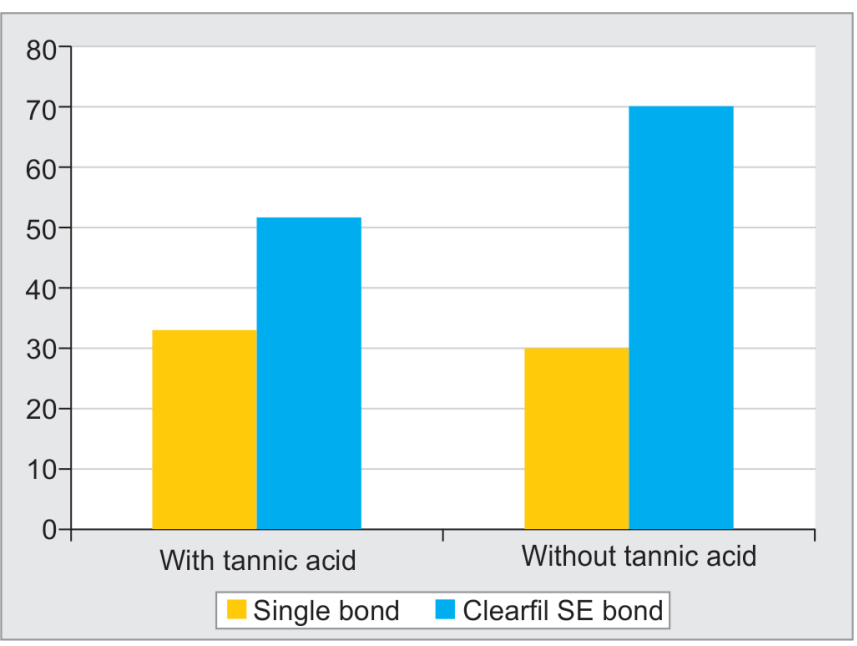

Graph 2: Mean of shear bond strength of SB and CSB subgroups with/without aging in TA group 
Table 3: Mean and standard deviation of shear bond strength in study groups

\begin{tabular}{llll}
\hline Groups & Type of material & With TA & Without TA \\
\hline Aging & SB & $33.38 \pm 21.41$ & $23.15 \pm 11.02$ \\
& CSB & $51.6 \pm 17.81$ & $71.03 \pm 21.75$ \\
\multirow{2}{*}{ No aging } & SB & $30.2 \pm 11.1$ & $63.01 \pm 23.75$ \\
& CSB & $70.68 \pm 16.23$ & $60.39 \pm 18.9$ \\
\hline
\end{tabular}

bond strength, and facilitation of the procedure. ${ }^{10}$ Over time, the bond strength of adhesives to dentin become weaker and would be less reliable and subject to breakdown. ${ }^{3,4}$ This is because of continuous masticatory forces and also the thermal stresses. As there are difference in thermal expansion coefficients in restorative materials and tooth structure, the bond degradation will occur. ${ }^{11-14}$

Several laboratory and clinical studies are indicated as sufficient evidences for the hydrolytic degradation of collagenous matrix by MMPs., ${ }^{6,7}$

We studied TA as a factor, which might enhance the bond strength through providing cross-links between collagen fibers. Bedran-Russo et al studied the various concentrations of TA as a cross-linker on the dentin of permanent teeth. They concluded that TA could cause excess of the dentinal stiffness. ${ }^{9}$ Also, their study showed that TA $(20 \%)$ could increase microtensile bond strength of adhesive SB. However, our findings indicated that TA reduced the SB strength and did not affect instant stability of CSB. This difference between the current study and Bedran-Russo ${ }^{9}$ findings could be explained by type of dentin and the time of TA application. Intertubular dentin of primary teeth is less than that of permanent teeth, and this might be the reason of lower bond strength of primary dentin to bondings. ${ }^{15}$ Another reason was the short time of TA application in the current study, while they applied it for 1 hour. When demineralized dentinal matrix was exposed to TA for 1 hour, the microtensile bond strength increased significantly.

Based on findings of the present study, the CSB adhesive possessed the highest bond strength among the studied groups. Correspondingly, two studies performed by Toledano and Jung et al indicated similar results. ${ }^{16,17}$

Several studies have introduced the TA as a protective agent. Tannic acid could cause incomplete elimination of the smear layer; it has a constriction effect on peritubular dentin and smear layer, which cause the closure of dentinal tubules and reduces irritant penetration into the pulp. ${ }^{18,19}$

Bitter et al showed that with TA usage stain penetrates less into dentinal tubules, which leads to pulp protection. ${ }^{20}$ Extended time treatment with TA might protect the collagen structures against the attack by phosphoric acid. ${ }^{9}$ Perhaps, despite our findings, if dentin is exposed to TA for a long duration, it would cause a significant elevation in the immediate bond strength of the SB adhesive. It has been noticed that using TA for about 1 hour is not practical in clinic and is done merely in laboratory studies.

\section{CONCLUSION}

As our findings indicate that there is no positive effect on using TA with SB or CSB adhesives in primary dentin, more researches are needed to be performed in order to understand the actual mechanisms of TA.

\section{ACKNOWLEDGMENTS}

The results presented in this study have been taken from a postgraduated thesis (no: 486). This study was supported by a grant from Mashhad University of Medical Sciences Research Council. Their financial aid is acknowledged.

\section{REFERENCES}

1. Ruschel HC, Ligocki GD, Flaminghi DL, Fossati AC. Microstructure of mineralized tissues in human primary teeth. J Clin Pediatr Dent 2011 Spring;35(3):295-300.

2. Dean, JA. McDonald and Avery's dentistry for the child and adolescent. Elsevier Health Sciences; 2015.

3. De Munck J, Van Meerbeek B, Yoshida Y, Inoue S, Vargas M, Suzuki K, Lambrechts P, Vanherle G. Four-year water degradation of total-etch adhesives bonded to dentin. J Dent Res. 2003;82(2):136-140.

4. Frankenberger R, Pashley DH, Reich SM, Lohbauer U, Petschelt A, Tay FR. Characterisation of resin-dentine interfaces by compressive cyclic loading. Biomaterials 2005 May;26(14):2043-2052.

5. Aranha AM, Giro EM, Hebling J, Lessa FC, Costa CA. Effects of light-curing time on the cytotoxicity of a restorative composite resin on odontoblast-like cells. J Appl Oral Sci 2010 Sep-Oct;18(5):461-466.

6. van Strijp AJ, Jansen DC, DeGroot J, ten Cate JM, Everts V. Host-derived proteinases and degradation of dentine collagen in situ. Caries Res 2003 Jan-Feb;37(1):58-65.

7. Pashley DH, Tay FR, Yiu C, Hashimoto M, Breschi L, Carvalho RM, Ito S. Collagen degradation by host-derived enzymes during aging. J Dent Res 2004 Mar;83(3):216-221.

8. Bedran-Russo AK, Pashley DH, Agee K, Drummond JL, Miescke KJ. Changes in stiffness of demineralized dentin following application of collagen crosslinkers. J Biomed Mater Res B Appl Biomater 2008 Aug;86(2):330-334.

9. Bedran-Russo AK, Yoo KJ, Ema KC, Pashley DH. Mechanical properties of tannic-acid-treated dentin matrix. J Dent Res 2009 Sep;88(9):807-811.

10. Nagi SM. Durability of solvent-free one-step self-etch adhesive under simulated intrapulpal pressure. J Clin Exp Dent 2015 Oct 1;7(4):e466-e470.

11. De Munck J, Van Landuyt K, Peumans M, Poitevin A, Lambrechts P, Braem M, Van Meerbeek B. A critical review of the durability of adhesion to tooth tissue: methods and results. J Dent Res 2005 Feb;84(2):118-132.

12. Erhardt MC, Toledano M, Osorio R, Pimenta LA. Histomorphologic characterization and bond strength evaluation of caries-affected dentin/resin interfaces: effects of long-term water exposure. Dent Mater 2008 Jun;24(6):786-798. 
13. Abdalla AI. Effect of long-term water aging on microtensile bond strength of self-etch adhesives to dentin. Am J Dent 2010 Feb;23(1):29-33.

14. Abdalla AI, Feilzer AJ. Four-year water degradation of a totaletch and two self-etching adhesives bonded to dentin. J Dent 2008 Aug;36(8):611-617.

15. Ricci HA, Sanabe ME, de Souza Costa CA, Pashley DH, Hebling J. Chlorhexidine increases the longevity of in vivo resin-dentin bonds. Eur J Oral Sci 2010 Aug;118(4):411-416.

16. Toledano M, Osorio R, Ceballos L, Fuentes MV, Fernandes CA, Tay FR, Carvalho RM. Microtensile bond strength of several adhesive systems to different dentin depths. Am J Dent 2003 Oct;16(5):292-298.
17. Jung YJ, Hyun HK, Kim YJ, Jang KT. Effect of collagenase and esterase on resin-dentin interface: a comparative study between a total-etch adhesive and a self-etch adhesive. Am J Dent 2009 Oct;22(5):295-298.

18. Sabbak SA, Hassanin MB. A scanning electron microscopic study of tooth surface changes induced by tannic acid. J Prosthet Dent 1998 Feb;79(2):169-174

19. Takahashi H, Okamoto Y, Fujinaka S, Shintani H. A pilot study of exposure of the smear layer to tannic acid solutions. J Prosthet Dent 1993 Sep;70(3):261-263.

20. Bitter NC. The effect of $25 \%$ tannic acid on prepared dentin: a scanning electron microscope-methylene blue dye study. J Prosthet Dent 1990 Jul;64(1):12-16. 